\title{
Introduction of a cartilage calcification insufficient rat as an infertile model animal
}

\author{
Minoru Watanabe ${ }^{1}$, Masami Tanaka ${ }^{2}$, Yuko Takeba $^{3}$, Masanori Ootaki ${ }^{3}$, Yuki Ohta $^{3}$, Taroh liri ${ }^{3}$, \\ Tsukasa Kobayashi $^{3}$, Daiki Watanabe ${ }^{3}$, Toshio Kumai ${ }^{4}$, Naoki Matsumoto ${ }^{3}$ \\ ${ }^{1}$ Institute for Animal Experimentation, St. Marianna University Graduate School of Medicine, Japan, ${ }^{2}$ Department of \\ Food and Nutrition, Junior College Division, The University of Aizu, Japan, ${ }^{3}$ Department of Pharmacology, St. \\ Marianna University School of Medicine, Japan, ${ }^{4}$ Department of Pharmacogenomics, St. Marianna University \\ Graduate School of Medicine, Japan
}

Rats with dwarfism accompanied by skeletal abnormalities, such as shortness of the limbs, tail, and body (dwarf rats), emerged in a Jcl-derived Sprague-Dawley rat colony maintained at the Institute for Animal Experimentation, St. Marianna University Graduate School of Medicine; they were named cartilage calcification insufficient (CCI, $c c i / c c i)$ rats (Experimental Animals, 64:121-128, 2015). The CCI characteristic associated with these dwarfisms show an autosomal recessive inheritance pattern. CCI rats with homozygous genes are infertile while apparently normal (N, cci/wt or $w t / w t)$ rats in this colony are fertile. The causes and mechanisms of infertility in CCI rats are still unknown, and clarifying these points may lead to the elucidation of causes and mechanisms of infertility in humans. Changes in the gene expression levels in the ovaries of 4-week-old CCI rats were examined by a microarray method and the ovarian gene expression in 14week-old CCI rats was examined by a real-time PCR method. Furthermore, ovarian tissue sections were subjected to HE staining and the serum gonadotropin and sex hormone levels were measured with an ELISA kit. The microarray analysis revealed several genes with altered expression levels in the 4-week old CCI rats, and a quantitative PCR was performed to investigate the expression of genes that are thought to be closely related to the ovarian function. As a result, the expression of the $3 \beta$-hydroxysteroid dehydrogenase ( $3 \beta$-HSD) gene in the CCI rat ovary (at 14 weeks of age) was found to be markedly lower than that of $\mathrm{N}$ rats. The serum estradiol and LH concentrations were significantly decreased in CCI rats, and the decrease of estradiol was presumed to be due to the decreased expression of $3 \beta$-HSD in the CCI rat ovary. Furthermore, the presence of numerous ovarian follicles was confirmed in CCI rat ovaries with a polycystic ovary - like morphology, and the presence of a corpus luteum could not be confirmed. The serum progesterone concentration also reduced in CCI rats, suggesting that the suppression of ovulation was occurring in CCI rats. The findings of the present study suggest that abnormalities in both steroidogenesis and ovulation have occurred in the ovaries of CCI rats. 